\title{
Clear Cell Sarcoma of the Kidney
}

National Cancer Institute

\section{Source}

National Cancer Institute. Clear Cell Sarcoma of the Kidney. NCI Thesaurus. Code C4264.

A rare pediatric sarcoma affecting the kidney. It is characterized by the presence of epithelioid or spindle cells forming cords or nests, separated by fibrovascular septa. It is associated with internal tandem duplications in the BCOR gene. It metastasizes to lung, bone, brain and soft tissue. 